\title{
Study of intraocular pressure and glaucoma risk in myopes and hypermetropes in middle aged adults
}

\author{
Samuel Cornelius Gnanadurai J. ${ }^{1}$, Vimala Karunanidhi S. ${ }^{2}$, Balaji Ramraj ${ }^{3}$, Sathish Kumar M. ${ }^{4}$, Swamyraj S.V. $^{5}$ \\ ${ }^{1}$ Dr. J. Samuel Cornelius Gnanadurai, Associate Professor, Department of Ophthalmology, ${ }^{2}$ Dr. S. Vimala Karunanidhi, \\ Senior Resident, Department of Ophthalmology, ${ }^{3}$ Dr. Balaji Ramraj, Associate Professor, Department of Community \\ Medicine, ${ }^{4}$ Dr. M. Sathish Kumar, Resident PG, Department of Ophthalmology, ${ }^{5}$ Dr. S.V. Swamyraj, Professor \& HOD, \\ Department of Ophthalmology, All Authors are affiliated with SRM Medical College Hospital and Research Centre, \\ Chennai, Tamil Nadu, India
}

Corresponding Author: Dr. J. Samuel Cornelius Gnanadurai, M.B.B.S., M.S. (ophthal), DNB, F.I.C.O., Associate Professor, Department of Ophthalmology, SRM Medical College Hospital and Research Centre, Chennai, Tamil Nadu, India. E-mail: samueldurai@yahoo.com

\begin{abstract}
Aim: To study the relationship between Intra ocular pressure and refractive errors (myopia and hypermetropia) and assess glaucoma risk in middle aged adults and compare with a normal emmetropic population of same age group. Materials and Methods: This is a prospective observational study of 150 patients between the age of 30-50 years attending ophthalmology outpatient department of SRM Medical College Hospital \& Research Centre for a period of 6 months. They were categorized into five groups as Emmetropia $(+0.5 \mathrm{D}$ to $-0.5 \mathrm{D})$, Hypermetropia( $>+0.5 \mathrm{D})$, Low Myopia (-0.75D to $<-3 \mathrm{D})$, Moderate Myopia (-3D to $-6 \mathrm{D})$ and High Myopia ( $>-6 \mathrm{D})$. Intraocular pressure will be measured with Goldmann applanation tonometer three times at one weekly interval and the average value will be taken. Results: The intra ocular pressure in moderate and high myopia was found to be higher compared to emmetropic, hypermetropic and low myopic patients which was statistically significant (P value-0.0001). In all the refractive error groups, the intra ocular pressure was higher in the 40-50 years age group compared to the 30-39 years age group. There was no statistically significant difference between Intra ocular pressure in the right eye and left eye and between the sexes in all the refractive error groups. Conclusion: The intra ocular pressure was higher in moderate and high myopia and in the 40-50 years age group. This confirms that intra ocular pressure increases with advancing age. Patients with moderate and high myopia have increased risk of developing Glaucoma. Therefore, it would be advisable to routinely check intra ocular pressure for myopes.
\end{abstract}

Key words: Glaucoma, Intra ocular pressure, Myopia, Refractive error.

\section{Introduction}

Refractive errors are one of the growing problems worldwide. Myopia is one of the commonest refractive errors with a worldwide prevalence of about $30 \%$ and up to $80 \%$ in Central Asian Population [1]. The average normal IOP in a human eye is $15.5 \mathrm{~mm} \mathrm{Hg}$ as seen in most population-based studies. Persistent rise in IOP of the eye leads to a complex condition called Glaucoma that may damage the optic nerve and lead to progressive blindness. Glaucoma is a leading cause of global irreversible blindness. Primary open angle glaucoma (POAG) is the most common form of glaucoma worldwide [2].

Manuscript received: $2^{\text {nd }}$ October 2019

Reviewed: $12^{\text {th }}$ October 2019

Author Corrected: $17^{\text {th }}$ October 2019

Accepted for Publication: $22^{\text {nd }}$ October 2019
There is a disproportionate burden of POAG in Asia, with Asians accounting for 53\% of POAG cases worldwide. Most population-based studies have found significant relation between intraocular pressure and myopia. This association is stronger in individuals with moderate to high myopia (>-3.00 dioptre) [3]. This may be because of longer axial length in myopia which may lead to weaker connective tissue support at the lamina cribrosa region of the optic nerve head. Scleral thinning associated with myopia may increase the susceptibility to raised intra ocular pressure, inducing further axial elongation of the eyeball and injury to the optic nerve head. Eyes with myopia seem to have a greater susceptibility for lamina cribrosa deformation due to raised intra ocular pressure leading to subsequent 
development of glaucoma. Also, intra ocular pressure induced strain may compress the lamina cribrosa and disrupt axonal transport of trophic factors which are essential for survival of retinal ganglion cells. Lamina cribrosa is the site where retinal ganglion cell axons congregate before traversing to the brain, therefore any mechanical strain at this site may initiate glaucomatous damage [4]. There are very few studies which have studied the relationship between intraocular pressure and hypermetropia. The risk of open angle glaucoma appears to increase with the degree of myopia [5]. Fluorescein angiographic studies have shown reduced blood flow in myopes compared to emmetropes and hypermetropes. The circulation to optic disc in myopes is also reduced and therefore myopes are more susceptible to raised intra ocular pressure. Glaucoma is a leading cause of blindness in people above 40 years. Most studies have shown that prevalence of glaucoma increases with age. The current study was done with the aim to study intraocular pressure changes in myopes and hypermetropes and compare with a normal emmetropic population in middle aged adults.

\section{Materials and Methods}

Aim of the study: To study the relationship between Intra ocular pressure and refractive errors (myopia and hypermetropia) and assess glaucoma risk in middle aged adults and compare with a normal emmetropic population of same age group.

Study Design: This is a prospective observational study of middle-aged patients attending ophthalmology outpatient department of SRM Medical College Hospital \& Research Centre. They were categorized into five groups as Emmetropia ( $+0.5 \mathrm{D}$ to $-0.5 \mathrm{D})$, Hypermetropia( $>+0.5 \mathrm{D})$, Low Myopia (-0.75D to $<-$ 3D), Moderate Myopia (-3D to $-6 \mathrm{D})$ and High Myopia(>-6D).

Sample size: 150 patients aged between $30-50$ years.

\section{Original Research Article}

Duration of study: 6 months

Diagnostic Methods: All the subjects will undergo a complete ocular examination including slit lamp examination, Intraocular pressure, dilated fundus examination, retinoscopy and refraction. BCVA (Best Corrected Visual acuity) will be assessed using Snellen's visual acuity chart. Intraocular pressure will be measured by Goldmann applanation tonometer by the same investigator in all cases. Intraocular pressure will be measured three times at one weekly interval and the average value will be taken. Patients with glaucoma risk will undergo Gonioscopy, Automated Perimetry and optic disc evaluation.

\section{Inclusion Criteria}

1. Age between 30 to 50 years

2. Emmetropes $(+0.5 \mathrm{D}$ to $-0.5 \mathrm{D})$

3. Hypermetropes $(>+0.5 \mathrm{D})$

4. Myopes $(>-0.75 \mathrm{D})$

\section{Exclusion Criteria}

1. Astigmatism $>1 \mathrm{D}$

2. Family history of Glaucoma

3. History of trauma or surgery in the eye

4. Patients on topical medications

5. Smokers/ alcoholics

6. Diabetes/ Hypertension

Statistical Analysis: The intraocular pressure in myopes, hypermetropes and emmetropes will be compared and statistically analyzed using the unpaired T test.

Ethics: Clearance was obtained from scientific and ethical committee of SRM Medical College Hospital and Research Centre for the study. Written and informed consent was obtained from all patients in English and local language. All data will be kept confidential.

\section{Results}

Table-6: Subgroups

\begin{tabular}{|c|c|c|}
\hline Group & No. of patients & \% \\
\hline Emmetropia & 40 & 26.66 \\
\hline Hypermetropia & 40 & 26.66 \\
\hline Low myopia & 30 & 20 \\
\hline Mod. myopia & 25 & 16.66 \\
\hline High myopia & 15 & 10 \\
\hline Grand Total & $\mathbf{1 5 0}$ & \\
\hline
\end{tabular}


A total of 150 patients took part in the study out of which 40 patients $(26.66 \%)$ were emmetropes $(+0.5 \mathrm{D}$ to $-0.5 \mathrm{D}), 40$ patients $(26.66 \%)$ had hypermetropic error $(>+0.5 \mathrm{D})$ and 70 patients $(46.66 \%)$ had myopic error. Among the myopes, 30 patients $(20 \%)$ were low myopes $(-0.75 \mathrm{D}$ to $<-3 \mathrm{D}), 25$ patients $(16.66 \%)$ were moderate myopes $(-3 \mathrm{D}$ to $-6 \mathrm{D})$ and 15 patients $(10 \%)$ were high myopes $(>-6 \mathrm{D})$.

Table-7: Comparison of IOP in right eye and left eye

\begin{tabular}{|c|c|c|c|c|c|c|}
\hline Group & RE mean & RE SD & LE mean & LE SD & T test & P value \\
\hline Emmetropia & 13.02 & 0.80 & 13.1 & 0.81 & -0.417 & 0.678 \\
\hline Hypermetropia & 14.07 & 0.82 & 14.05 & 0.78 & 0.139 & 0.890 \\
\hline Low myopia & 14.1 & 0.80 & 14 & 0.83 & 0.474 & 0.637 \\
\hline Mod. myopia & 16.12 & 0.78 & 16.24 & 0.77 & -0.544 & 0.589 \\
\hline High myopia & 18.46 & 1.12 & 18.6 & 1.12 & -0.325 & 0.748 \\
\hline
\end{tabular}

There was no statistically significant difference between Intra ocular pressure in the right eye and left eye in all the refractive error groups.

Table-8: Co-relation of refractive status and IOP

\begin{tabular}{|c|c|c|c|}
\hline Group & No. patients & IOP mean & IOP SD \\
\hline Emmetropia & 40 & 13.06 & 0.77 \\
\hline Hypermetropia & 40 & 14.06 & 0.74 \\
\hline Low myopia & 30 & 14.05 & 0.78 \\
\hline Mod. myopia & 25 & 16.18 & 0.73 \\
\hline High myopia & 15 & 18.53 & 1.08 \\
\hline Grand Total & $\mathbf{1 5 0}$ & $\mathbf{1 4 . 5 9}$ & $\mathbf{1 . 8 4}$ \\
\hline
\end{tabular}

ANOVA - 165.856, P - 0.0001

The intra ocular pressure in moderate and high myopia was found to be higher compared to emmetropic, hypermetropic and low myopic patients which was statistically significant (P value-0.0001). As the grade of myopia increases, the intra ocular pressure was found to increase in our study, especially moderate and high myopes were found to have significantly higher intra ocular pressures thereby increasing the risk of developing glaucoma.

Table-9: Co-relation of IOP with age

\begin{tabular}{|c|c|c|c|c|c|}
\hline Group & Age & IOP mean & IOP SD & T test & $P$ value \\
\hline Emmetropia & $30-39$ & 12.75 & 0.53 & -2.225 & 0.032 \\
\hline Emmetropia & $40-50$ & 13.27 & 0.84 & & \\
\hline Hypermetropia & $30-39$ & 14 & 0.80 & -0.386 & 0.702 \\
\hline Hypermetropia & $40-50$ & 14.09 & 0.73 & & \\
\hline Low myopia & $30-39$ & 13.73 & 0.59 & -2.137 & 0.041 \\
\hline Low myopia & $40-50$ & 14.29 & 0.83 & & \\
\hline Mod. myopia & $30-39$ & 15.95 & 0.68 & -1.325 & 0.198 \\
\hline Mod. myopia & $40-50$ & 16.33 & 0.76 & & \\
\hline High myopia & $30-39$ & 18 & 0.71 & -1.662 & 0.120 \\
\hline High myopia & $40-50$ & 18.88 & 1.20 & & \\
\hline
\end{tabular}

In all the refractive error groups, the intra ocular pressure was higher in the 40-50 years age group compared to the 30-39 years age group. This confirms that intra ocular pressure increases with advancing age. 
Original Research Article

Tabl-10: Co-relation of IOP with gender

\begin{tabular}{|c|c|c|c|c|c|}
\hline Group & Sex & IOP mean & IOP SD & T test & P value \\
\hline Emmetropia & $\mathrm{M}$ & 13 & 0.87 & -0.541 & 0.592 \\
\hline Emmetropia & $\mathrm{F}$ & 13.13 & 0.64 & & \\
\hline Hypermetropia & $\mathrm{M}$ & 14.19 & 0.60 & 1.148 & 0.258 \\
\hline Hypermetropia & $\mathrm{F}$ & 13.92 & 0.87 & & \\
\hline Low myopia & $\mathrm{M}$ & 14.11 & 0.90 & 0.552 & 0.585 \\
\hline Low myopia & $\mathrm{F}$ & 13.96 & 0.59 & & \\
\hline Mod. myopia & $\mathrm{M}$ & 16.10 & 0.64 & -0.563 & 0.579 \\
\hline Mod. myopia & $\mathrm{F}$ & 16.27 & 0.86 & & \\
\hline High myopia & $\mathrm{M}$ & 18 & 0.59 & -1.970 & 0.070 \\
\hline High myopia & $\mathrm{F}$ & 19 & 1.20 & & \\
\hline
\end{tabular}

In all the refractive error groups, there was no statistically significant difference in the intra ocular pressure between males and females.

\section{Discussion}

Refractive errors are one of the most common ocular problems especially in younger individuals. Myopia has emerged as a major health issue worldwide, and its prevalence has increased rapidly in the past few decades. There is an increased prevalence of glaucoma among myopes compared to hypermetropes and emmetropes.

There are several hypotheses to explain raised intra ocular pressure in myopia. High myopes with increased axial length may be associated with scleral thinning, poor elasticity of the sclera wall and are more vulnerable to sclera tension across the optic nerve head and lamina cribrosa. The presence of raised intra ocular pressure in high myopic eyes is likely to further induce mechanical strain on the vulnerable optic nerve head and lamina cribrosa, thereby leading to compression of retinal ganglion cells followed by axonal damage [5].

There is increased stress on the global wall and decreased ocular rigidity in myopic eyes which may lead to raised intra ocular pressure. The retinal nerve fiber layer (RNFL) thickness has been found to be reduced in myopia which increases the chances of developing glaucoma. High myopes are more likely to be steroid responders which increases the chances of raised intra ocular pressure [6].

It is postulated that the ciliary body is relatively posterior in relation to canal of schlemm in myopic eyes, therefore it has less chances of widening the trabecular spaces during accommodation. The circulation to optic disc and choroidal blood flow is reduced in myopes which make them susceptible to raised intra ocular pressure. Most population-based studies have suggested that moderate to high myopia is associated with primary open angle glaucoma (POAG) and ocular hypertension. In the Blue Mountains Eye study [7], eyes with moderate myopia were 2 times more likely to have primary open angle glaucoma (POAG). In the Barbados Eye study [8], a myopic refractive status was one of several risk factors for primary open angle glaucoma (POAG) in adult black population.

The Beijing Eye study [9] found significant relationship between moderate to high myopia and glaucoma. The Singapore Malay Eye study [10] showed an association of moderate myopia and increasing axial length with primary open angle glaucoma (POAG) independent of other factors, including central corneal thickness.

Also, a shorter anterior chamber depth was associated with primary open angle glaucoma (POAG), probably because anterior chamber was shallower in older persons because of increased lens thickness. Jin A. Choi et al [11] found that myopic refractive error was an independent predictor of higher intra ocular pressure in non-glaucomatous eyes, and the association between refractive error and intra ocular pressure differed according to age.

Myopia appears to affect intra ocular pressure in young to middle aged subjects, whereas age-related risk factors such as body mass index (BMI), waist circumference, diabetes, hypertension have a greater influence on intra ocular pressure in old-age subjects. Elevated intra ocular pressure in older post-menopausal women is speculated to be due to the sympathetic activity stimulated by increased insulin resistance and decreased estrogen action on the inflow and outflow facility. YihChung Tham et al [12] found that eyes with higher intra ocular pressure $(>20 \mathrm{mmHg})$, longer axial length 
$(>25.5 \mathrm{~mm})$ and moderate to high myopia (<-3.0 Dioptres) were independently associated with primary open angle glaucoma (POAG). Eyes with high intra ocular pressure and moderate to high myopia had approximately 4.5 - fold increased risk of primary open angle glaucoma (POAG) compared to eyes without myopia and with lower intra ocular pressure. Eyes with high intra ocular pressure and axial length of $>25.5 \mathrm{~mm}$ had approximately 16-fold increased risk of primary open angle glaucoma (POAG). Roopa Somashekar Mathapathi et al [13] studied 120 cases of refractive error aged between 20 to 60 years and compared with emmetropic eyes.

There was a significant correlation between high myopia and raised intraocular pressure but there was no correlation between low myopia and hypermetropia with intraocular pressure. A study by Joseph D.S et al [14] of 100 patients found that there was a statistically significant correlation between intra ocular pressure and myopia in the moderate and high myopia groups. Dudhabhate Anil et al [15] studied 50 patients and found higher degrees of myopia as an important risk factor for ocular hypertension.

A total of 150 patients took part in our study out of which 40 patients $(26.66 \%)$ were emmetropes $(+0.5 \mathrm{D}$ to $-0.5 \mathrm{D}), 40$ patients $(26.66 \%)$ had hypermetropic error $(>+0.5 \mathrm{D})$ and 70 patients $(46.66 \%)$ had myopic error. Among the myopes, 30 patients $(20 \%)$ were low myopes $(-0.75 \mathrm{D}$ to $<-3 \mathrm{D}), 25$ patients $(16.66 \%)$ were moderate myopes $(-3 \mathrm{D}$ to $-6 \mathrm{D})$ and 15 patients $(10 \%)$ were high myopes(>-6D).

The intra ocular pressure in moderate and high myopia was found to be higher compared to emmetropic, hypermetropic and low myopic patients which was statistically significant ( $\mathrm{P}$ value-0.0001). As the grade of myopia increases, the intra ocular pressure was found to increase in our study, especially moderate and high myopes were found to have significantly higher intra ocular pressures thereby increasing the risk of developing glaucoma. Therefore, it would be advisable to routinely check intra ocular pressure for myopes.

Though Goldmann applanation tonometer is the gold standard for measuring intra ocular pressure, it is a contact procedure with higher chances of developing corneal infections. Therefore, Non-contact tonometer [16] can be considered as a screening tool to check intra ocular pressure for myopes as it is easy to perform and non-invasive. In all the refractive error groups, the intra ocular pressure was higher in the 40-50 years age group compared to the 30-39 years age group. This confirms that intra ocular pressure increases with advancing age. Therefore, routine intra ocular pressure measurement should be done for all individuals above 40 years. There was no statistically significant difference between Intra ocular pressure in the right eye and left eye in all the refractive error groups. In all the refractive error groups, there was no statistically significant difference in the intra ocular pressure between males and females.

The limitation of this study would be that corneal thickness, corneal curvature and axial length were not measured. These factors may influence the correlation between refractive error, intra ocular pressure and biomechanical properties of cornea [17].

\section{Conclusion}

The intra ocular pressure in moderate and high myopia was found to be higher compared to emmetropic, hypermetropic and low myopic patients which was statistically significant. In all the refractive error groups, the intra ocular pressure was higher in the 40-50 years age group compared to the 30-39 years age group. This confirms that intra ocular pressure increases with advancing age.

Therefore, routine intra ocular pressure measurement should be done for all individuals above 40 years. In all the refractive error groups, there was no statistically significant difference in the intra ocular pressure between males and females. There was no statistically significant difference between Intra ocular pressure in the right eye and left eye in all the refractive error groups. Patients with moderate and high myopia have increased risk of developing Glaucoma.

Therefore, it would be advisable to routinely check intra ocular pressure for myopes. Though Goldmann applanation tonometer is the gold standard for measuring intra ocular pressure, it is a contact procedure with higher chances of developing corneal infections. Therefore, Non-contact tonometer can be considered as a screening tool to check intra ocular pressure for myopes.

\section{What the study adds to the existing knowledge?}

Glaucoma is a leading cause of blindness in middle aged and elderly population. Early diagnosis of Glaucoma is the key to effective management and prevention of blindness. IOP is an established risk factor for glaucoma and is the only modifiable risk factor for progression of glaucoma. This study has 
shown that patients with moderate and high myopia have higher intra ocular pressure compared to emmetropes, and therefore have increased risk of developing Glaucoma. Therefore, it would be advisable to routinely check intra ocular pressure for myopes. Non-contact tonometer can be considered as a screening tool to check intra ocular pressure for myopes, as it is easy to perform and non-invasive.

\section{Author's Contributions}

Dr. J. Samuel Cornelius Gnanadurai: Concept, study design, Scientific and ethical committee presentation, data collection, data summarization and interpretation, manuscript preparation and manuscript review.

Dr. S. Vimala Karunanidhi: Scientific and ethical committee presentation, patient selection and data collection.

Dr. Balaji Ramraj: Statistical analysis.

Dr. M. Sathish Kumar: Data Collection

Dr. S.V. Swamyraj: Data Collection and Proof approval.

\section{Funding: Nil, Conflict of interest: Nil}

Permission from IRB: Yes

Ethical approval: This study was approved by the Institutional Ethics Committee.

\section{References}

1. Tham YC, Li X, Wong TY, Quigley HA, Aung T, Cheng CY. Global prevalence of glaucoma and projections of glaucoma burden through 2040: a systematic review and meta-analysis. Ophthalmol. 2014;121(11):2081-2090. doi: 10.1016/j.ophtha. 2014 05.013. Epub 2014 Jun 26.

2. Wong TY, Klein BE, Klein R, Knudtson M, Lee KE. Refractive errors, intraocular pressure, and glaucoma in a white population. Ophthalmol. 2003;110(1):211-217. doi: 10.1016/s0161-6420(02)01260-5.

3. Lee AJ, Saw SM, Gazzard G, Cheng A, Tan DT. Intraocular pressure associations with refractive error and axial length in children. $\mathrm{Br} \mathrm{J}$ Ophthalmol. 2004;88(1):5-7. doi: 10.1136/bjo.88.1.5.

4. Grødum K, Heijl A, Bengtsson B. Refractive error and glaucoma. Acta Ophthalmol Scand. 2001;79 (6):560-566. doi: 10.1034/j.1600-0420.2001.790603.x.

\section{Original Research Article}

5. Wu SY, Nemesure B, Leske MC. Glaucoma and myopia. Ophthalmol. 2000;107(6):1026-1027. doi: 10.1016/s0161-6420(00)00051-8.

6. Vijaya L, Rashima A, Panday M, Choudhari NS, Ramesh SV, Lokapavani V, et al. Predictors for incidence of primary open-angle glaucoma in a South Indian population: the Chennai eye disease incidence study. Ophthalmol. 2014;121(7):1370-1376. doi: 10.1016/j.ophtha.2014.01.014. Epub 2014 Mar 18.

7. Mitchell P, Hourihan F, Sandbach J, Wang JJ. The relationship between glaucoma and myopia: the Blue Mountains Eye Study. Ophthalmol. 1999;106(10):20102015. doi: 10.1016/s0161-6420(99)90416-5.

8. Leske MC, Wu SY, Hennis A, Honkanen R, Nemesure B; BESs Study Group. Risk factors for incident open-angle glaucoma: the Barbados Eye Studies. Ophthalmol. 2008;115(1):85-93. Epub 2007 Jul 16. doi: 10.1016/j.ophtha.2007.03.017.

9. Xu L, Wang Y, Wang S, Wang Y, Jonas JB. High myopia and glaucoma susceptibility the Beijing Eye Study. Ophthalmol. 2007;114(2):216-220. Epub 2006 Nov 21. doi: 10.1016/j.ophtha.2006.06.050.

10. Perera SA, Wong TY, Tay WT, Foster PJ, Saw SM, Aung T. Refractive error, axial dimensions, and primary open-angle glaucoma: the Singapore Malay Eye Study. Arch Ophthalmol. 2010;128(7):900-905. doi: 10.1001/ archophthalmol.2010.125.

11. Choi JA, Han K, Park YM, Park CK. Age-related association of refractive error with intraocular pressure in the Korea National Health and Nutrition Examination Survey. PLoS One. 2014;9(11):e111879. doi: 10.1371/ journal.pone.0111879. eCollection 2014.

12. Tham YC, Aung T, Fan Q, Saw SM, Siantar RG, Wong TY, et al. Joint effects of intraocular pressure and myopia on risk of primary open-angle glaucoma: the Singapore epidemiology of eye diseases study. Scientific reports. 2016;6:19320. doi: 10.1038/srep 19320.

13. Roopa Somashekar Mathapathi et al. Association of refractive errors with intra ocular pressure and its relationship with age and gender. Ind J Clinic Anat Physiol. 2016;3(4):419-422. doi:10.5958/2394-2126. 2016.00095 .5

14. Joseph DS, Thampi B, Joosadima A, Mohan A. A study on association between intraocular pressure and 
myopia. Int J Res Med Sci. 2016;4(6):2202-2205. http://dx.doi.org/10.18203/2320- 6012. ijrms20161786.

15. Anil D, Nitnavre M. A study on correlation between intra ocular pressure and myopia. Int $\mathrm{J}$ Sci Res .2018;7(6): 3-4.

16. Lin HY, Hsu WM, Chou P, Liu CJ, Chou JC, Tsai $\mathrm{SY}$, et al. Intraocular pressure measured with a noncontact tonometer in an elderly Chinese population: the Shihpai Eye Study. Arch Ophthalmol. 2005;123(3): 381-386. doi: 10.1001/archopht.123.3.381

17. Lim L, Gazzard G, Chan YH, Fong A, Kotecha A, Sim EL, et al. Cornea biomechanical characteristics and their correlates with refractive error in Singaporean children. Investigat Ophthalmol Vis Sci. 2008;49 (9): 3852-3857. doi: 10.1167/iovs.07-1670.

\section{How to cite this article?}

Samuel Cornelius Gnanadurai J., Vimala Karunanidhi S., Balaji Ramraj, Sathish Kumar M., Swamyraj S.V. Study of intraocular pressure and glaucoma risk in myopes and hypermetropes in middle aged adults. Trop J Ophthalmol Otolaryngol.2019; 4(6):388-394. doi:10.17511/jooo.2019.i06.06 